\title{
Robust sub-50 fs cavity-laser phase stabilization for ultra- fast electron diffraction instruments
}

\author{
Martin R. Otto ${ }^{1}$, Laurent P. René de Cotret $^{1}$, Mark J. Stern ${ }^{1}$, and Bradley J. Siwick ${ }^{1,2, *}$ \\ ${ }^{1}$ Department of Physics, Center for the Physics of Materials, McGill University, 3600 University Street, \\ Montreal, Quebec, Canada H3A 2T8 \\ ${ }^{2}$ Department of Chemistry, McGill University, 801 Sherbrooke Street W, Montreal, Quebec, Canada \\ H3A 0B8
}

\begin{abstract}
A novel laser-RF cavity synchronization scheme was developed for ultrafast electron diffraction instruments. The approach directly generates a synchronized microwave tone by retrieving a phase-locked high-harmonic by photodetection. The radio-frequency cavity which performs electron pulse compression is detuning compensated using an active phase stabilization loop. Timing stability improved from 100 fs to 5 fs RMS and long-term time-zero stability improved to below our measurement resolution of 50 fs determined by pulse streaking.
\end{abstract}

\section{Introduction}

Unmodified by external fields, space-charge dynamics in ultrashort electron pulses result in a trade-off between pulse fluence and time resolution that is detrimental to ultrafast electron diffraction and imaging experiments. Microwave compression, a technique that compensates for space-charge driven pulse broadening, has been demonstrated to be very effective in the single shot limit yielding electron pulses below $100 \mathrm{fs}$ at $100 \mathrm{keV}$ [1]. Unfortunately, the stability of the cavity-laser synchronization systems that have been employed to date are insufficient to provide pulse duration limited time-resolution in ultrafast electron diffraction instruments over the several hours required to acquired a full data-set. As a result of this instability, the primary benefit of microwave pulse compression has been an increase in bunch charge rather than a dramatic improvement in time resolution.

In this work we demonstrate a new approach [2] to laser-cavity synchronization and stabilization that provides electron pulse duration limited time-resolution over $>10 \mathrm{hrs}$, effectively solving the jitter-problem in RF compressed UED experiments. The first step of our approach is the passive generation of a harmonic phase-locked $3 \mathrm{GHz}$ signal via photo-detection of the oscillator laser pulse train followed by continuous-wave low phase-noise filtering and amplification sufficient for electron pulse compression in a $\mathrm{TM}_{010}$ cavity. The second step is the retrieval of this signal from the cavity using an in-situ antenna for use in an active synchronization enhancement system that measures and compensates for phase fluctuations arising from a range of environmental and amplifier instabilities. Together these lead to a greater than ten-fold improvement in laser-microwave synchronization. We further demonstrate that

\footnotetext{
*e-mail: bradley.siwick@mcgill.ca
} 

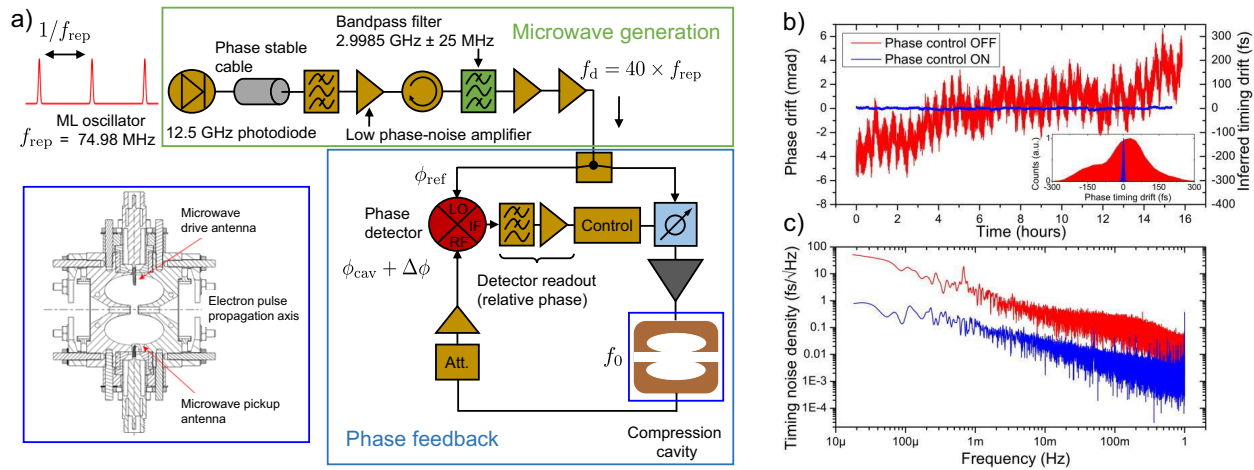

Figure 1. a) Schematic of synchronization system: The oscillator laser pulse train is incident on a high-bandwidth photodiode and the $\sim 3 \mathrm{GHz}$ harmonic of the repetition rate is generated, filtered and amplified. The signal is split, one half serving as a phase-stable reference and the other is amplified further for electron pulse compression in a $\mathrm{TM}_{010}$ compression cavity. Signal transmitted through the cavity is mixed with the reference and the relative phase fluctuations are measured and used for active phase feedback. b) Electronic phase timing measurement of the microwave compression system for the instrument described in [2]. Data for feedback off (red) and on (blue) are compared over the course of several hours and histograms of the phase fluctuations are shown in the inset. c) Additive timing noise power spectral density of the data shown in $b$ ).

this system improves the long-term stability of pump-probe time-delay and the temporal impulse response function of the UED instrument by correcting for phase noise over a very broad low-frequency band. This improved performance is due to the elimination of several sources of phase instability inherent in previous approaches; i) amplitude-phase modulations errors in generation of microwave signal using phase-locked loops, ii) Phase instability in power amplification iii) Phase drift in the cavity response due to thermal induced frequency detuning [2]. We expect these results will contribute to the advancement of ultrafast electron diffraction and imaging instruments.

\section{Results and Discussion}

We determined the cavity phase noise that remains in this system by mixing the cavity antenna/pickup loop signal with a pre-compression reference. The electronic phase stability measurements are shown in Fig. 1 b) and c). We find the additive phase noise power spectral density decreased by nearly two decades over the detection frequency range. We also directly measure the change in the arrival time of the electron pulses with respect to a femtosecond optical pump pulse using an optically triggered $10 \mathrm{GHz}$ streak camera similar to that presented in Ref. [3]. The impulse response function (IRF) is determined for a pulse charge of $0.2 \mathrm{pC}$ as a function of the compression signal phase. At each phase value, 30 pictures at $1 \mathrm{~s}$ exposure and $1 \mathrm{kHz}$ repetition rate time are collected $\left(3 \times 10^{4}\right.$ shots). The IRF as a function of phase is shown in Fig. 2 c). We find that the IRF approaches $50 \mathrm{fs}$ for optimal power and phase, at which point the measurement is limited by the temporal resolution of the streak camera. We measure an upper limit IRF of $\tau_{e}=45 \pm 2 \mathrm{fs}$ (106 fs full-width at half-maximum). We note that this is an improvement by over a factor of 3 when compared to previously reported instrument performance [4]. 

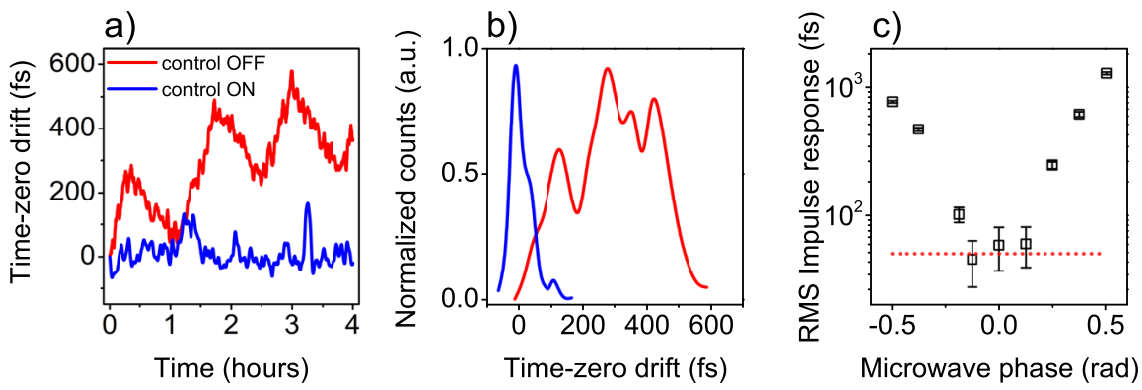

Figure 2. a) Electron pulse arrival time measured with phase feedback control both off (red) and on (blue). b) Histograms of the traces shown in a) depicting the long-term stability of time-zero. c) The temporal impulse response function of the UED instrument as a function of phase for a bunch charge of $0.2 \mathrm{pC}$ and cavity power of $44.7 \mathrm{~W}$. The dashed red line represents the time-resolution of the streak camera.

\section{Conclusion}

We have achieved ultrafast electron diffraction with pulse compression using phase-locked microwaves synthesized directly from a mode-locked oscillator. Furthermore, we have designed and characterized a high-performance integrated microwave phase feedback system which compensates for detuning induced phase fluctuations in compression cavities improving the laser-microwave synchronization level by a factor of 10 to below 5 fs RMS. The long-term arrival time stability of the electron pulse is also improved from $>200$ fs to below our measurement resolution of $<50$ fs RMS with phase stabilization. The impulse response function of the instrument is measured to be less than 70 fs RMS over many hours. Our results illustrate that high-brightness UED instruments can be realized in a simpler approach and pushed to a regime where time-resolution is limited by the duration of the compressed electron pulse, not synchronization quality.

\section{References}

[1] T. van Oudheusden, P. L. E. M. Pasmans, S. B. van der Geer, M. J. de Loos, M. J. van der Wiel, and O. J. Luiten. "Compression of subrelativistic space-charge-dominated electron bunches for single-shot femtosecond electron diffraction". Phys. Rev. Lett., 105, 264801, (2010).

[2] M. R. Otto, L. P. Ren de Cotret, M. J. Stern, and B. J. Siwick. "Solving the jitter problem in microwave compressed ultrafast electron diffraction instruments: Robust sub-50 fs cavity-laser phase stabilization". Struc. Dyn., 4:5, 051101, (2017) and references therein.

[3] G. H. Kassier, K. Haupt, N. Erasmus, E. G. Rohwer, H. M. von Bergmann, H. Schwoerer, S. M. M. Coelho, and F. D. Auret. "A compact streak camera for $150 \mathrm{fs}$ time resolved measurement of bright pulses in ultrafast electron diffraction". Rev. Sci. Inst., 82, 105103, (2010).

[4] Robert P. Chatelain, Vance R. Morrison, Chris Godbout, and Bradley J. Siwick. "Ultrafast electron diffraction with radio-frequency compressed electron pulses". Appl. Phys. Lett., 101,081901, (2012). 